\title{
The Role of Social Networking on Adaptability and Competitive Advantage: An Empirical Evidence from Small and Medium-sized Enterprises in Restaurant Industry, Surabaya, Indonesia
}

\author{
Endo Wijaya Kartika ${ }^{1, *}$ and Thomas Stefanus Kaihatu ${ }^{2}$ \\ ${ }^{1}$ Department of Management, Faculty of Business and Economics, Petra Christian University, \\ J1. Siwalankerto No. 121-131, Surabaya 60236, Indonesia. \\ ${ }^{2}$ Department of Management, Faculty of Management and Business, Ciputra University, \\ CitraLand CBD Boulevard, Surabaya 60219, Indonesia.
}

\begin{abstract}
Business competition that occurs in every industry in the world these days is totally inevitable, especially in Indonesia as one of the new emergent market in the world. Every companies are expected to be able to cope and compete well to achieve the best result for the shake of the companies' future. However, Small and Medium-sized Enterprises (SME) often encounter so many problems during their competition which could impacted on the decreasing company performance and to some extend the worse result which is bankruptcy. This research is conducted to investigate on the existence of gap that occur due to the lack of empirical evidence that focus on social networking, adaptability and competitive advantage on SMEs in Indonesia. Thus, this research focuses on the leader's social networking and organizational adaptability to support the creation of competitive advantage. This is an associative causal research using 30 restaurant owners in Surabaya city as the respondent which has a maximum number of employees of 20 peoples. Structural Equation Modelling (SEM) is used to answer the research questions. It is found out that social networking affects significantly on adaptability, while adaptability affects on competitive advantage insignificantly.
\end{abstract}

Keywords: Business competition, business environment, networking collaboration, prominent advantages, strategic management.

\section{Introduction}

Business competition that occurs in every industry in the world these days is totally inevitable, especially in Indonesia as one of the new emergent market in the world. Every companies in their industries are expected to be able to cope and compete well to achieve the best result for the shake of their companies' future. One of the main requirements that must be done by each of them is to create the best performance as the most prominent

\footnotetext{
*Corresponding author: endo@petra.ac.id
} 
measurement for the company's success indicator especially in both achieving company's vision and mission, and also in showing efforts in competing with its competitors in the same industry.

One essential consideration that should be considered in the context of competition is the scale of each competing company, which is often a larger company which should be far more enable to compete with its competitors, while a smaller company which will tend to struggle in order to survive. Therefore, each company should have obliged to find the most prominent advantages, in order to win and survive.

Small and Medium-sized Enterprises (SME) often encounter so many problems during their competition which could impacted on the decreasing company performance and to some extend the worser result which is bankruptcy. Despite of the important role in regional and national economic growth, few studies were conducted in SME context to understand and also to provide solution for these companies, especially in strategic management studies context. Mostly it happened due to stereotyping that considered SME as a collection of companies that fail to grow into a bigger company, relying on oldfashioned management approaches, or even without any strategy, planning, nor analysis at all. Thus, major factors that dominate the characteristics of SME are the lacking of resources to compete, although most of these companies tend to have a higher level of flexibility and uniqueness to provide a distinctive capability that considered as a competitive advantage [1].

In the past few years, reviews related to competitive advantage concept were considered increasing and developing rapidly, due to the effort to understand competitive advantage concept in different context. One of the prominent with a different view was the thought of Reeves \& Deimler [2], which emphasized on adaptability as a new competitive advantage. Despite of its commonality in term and it is not considered as something new, adaptability concept of thought currently could be considered as the basis for leaders to maintain and to remain competitive. This concept leads to the importance of capability in company to think and to be innovative in fast-paced manner, especially in uncertainty conditions which lead to a more strategically challenging for the company. Company's ability to adapt and change its resources is considered as one of the most important ways to be able to provide a competitive advantage and will also lead to better company performance.

In broadly manner, the concept of thought of adaptability views that traditional strategic perspective with a static view is no longer able to provide optimal results, therefore a new capability should be needed to be able to adapt more quickly. Common analogy to describe this condition that a company is expected to be able to learn better and deeply about how to do something new, rather than being able to do certain things well [3]. Therefore, this concept tends to run in dynamic and uncertain environments so that the strategic decision making process needs to be reactive and experimental. This point of view in term of theory which a company tends to emphasize its ability to adapt to dynamic market changes can be described as dynamic capability. Hence, continuous adaptation and evolution of working systems will support a better performance which considered as the adaptation capability will also result in the increasing of company performance [4].

In actual reality, what is really happened these days is actually not the strongest or the first will definitely survive, but the first who can adapt well will win survive in the end [5]. Principally, this statement could provide additional support in SMEs context that usually meet challenging problems and constraints during their competition. However, from research perspective there is still lacking of research domains that focus on SMEs, especially related to adaptability concept, and even empirical research as evidence based.

Therefore, this research was conducted to provide an empirical evidence regarding the adaptability capability by SME especially in Indonesia, whereas could be said that SME that exist these days are so many and could be the source of support toward a nation's 
economic growth and sustainability. In Indonesia, the term of SME is considered as UMKM (Micro, Small and Medium Enterprises), which are generally distinguished by the amount of wealth and annual sales. These companies in Indonesia also have to conduct business competition with both another SMEs and large scale companies in various industries.

Competitive condition also occurs in restaurant industry in Indonesia, which specifically occurs in the city of Surabaya. This condition is considered as the effect of the attractiveness of business in this industry that looks promising. The growth of the restaurant business in Surabaya can be said to be quite significant and thus, makes more new participants as new restaurants emerge from domestic and also international which attracts foreign investment as the impact from the existence of Asean Economic Community (AEC), which began in late 2015 [6].

These conditions also make the existing restaurant players become more anxious, since there are more players imply for more rapid competition which leads to survival for the fittest. These days, restaurant business in Surabaya city tend to be dominated by SMEs which have quite a lot of limitations in expanding or even competing directly with their competitors in general and foreign investment restaurants in particular. Therefore, every restaurant should conduct their best performance by showing their competitive advantages.

This concept could be interpreted as how a company posits itself in a business environment that interacts with other stakeholders within a business process, which also leads to the company's attitude toward other companies whether as competitors or collaborators that can collaborate together to create a better cooperation and benefits. Hence, based on this concept, SMEs can actually use the opportunity which lead to cooperation and networking that will be useful both present and the future. Previous studies emphasize on the role of networking, especially for SMEs which could contribute to creation of better performance [7]. The concept of networking itself is principally inherent to a leader who is often personally involved in interaction with other parties. Therefore, in SMEs, owners and concurrently also as leaders must be able to bring their company into a much larger network to increase its competitive advantages.

This research was conducted to investigate on the existence of gap that occured due to the lack of empirical evidence that focus on networking and adaptability on SMEs in Indonesia. Thus, this research focuses on the SMEs adaptability that support the creation of competitive advantages which influenced by networking capabilities of leaders to create a better performance.

\section{Literature review}

\subsection{Social networking}

Networking collaboration is considered as one of the most important things in business, which is caused by the need of network connection by most of companies to access their resources needed at less or no cost required. Networking could help in developing companies' credibility, targeting new markets, consumers, and suppliers which encourages innovation and build strategic cooperative partnerships. Business could form a good relationship with various organizations which could then influence the success or failure of its performance both directly and indirectly. The company should depend on its capabilities, as the ability to match the external capabilities with its requirement. To collaborate with many people or different organizations, it does not always require to be connected in a formal structure, nevertheless it should able to create interdependencies between one another. Business owner sometimes does not have all the insights and 
capabilities to make the company grow. Thus, networking is required to be able to find experts who could fill the gaps that the owner does not have, especially to create an important role to gain a strong networking capability with its environment. The definition of networking does not seem too clear and has not been well defined, as stated by Ford \& Mouzas [8] that business networking is a clear effort from an actor to change or to build the process of interaction or relationship structure that related with its environment directly or indirectly.

In a business environment, heterogeneity assumptions or diversity of attitudes and behaviors of a consumer groups or suppliers is important to understand. Noting that it could create interdependencies which could lead to form business networking. Thus, the role of management is to contribute to the creation of mutualism between company and consumers, suppliers, or even the other stakeholders. Therefore, cooperation relationships could appear to complement each other in the form of occurred interactions. Networking could be seen from the interaction perspective that emphasize on the importance of an inseparable chain of network implications in business process management, and this could lead to some reasons. Firstly, it is interesting to note that because interaction is a process that occurs between actors so that no actor has the freedom or free resources to implement independently without dealing or collaborating with other actors. Thus, networking is important as a base to create mutualism between actors; Secondly, the interaction process in business in not only limited to simple communication or negotiation, but substantially becomes prominent due to adjust the needs between the actors, which could also lead to adaptation or transformation of resources between the actors. Thus, networking is important to transform a complex dependency between actors; Thirdly, networking dependencies that involves external activities could lead to a greater influence by the actors and their resources to control the business. Fourth, business interaction process should be a continuous process that also requires long-term approach by the management.

\subsection{Adaptability}

In general, adaptability could be defined as the ability of a company to react quickly to opportunities and risks and convert it become a business advantage [8]. Adaptability could lead to some positive impact including, Company's capacity to respond to consumer needs; The ability to make optimal choices related to responses to change based on the information related to business environment in the past, present, and future; The recognition of main people who could adapt and encourage to take reasonable risks and also the ability to build new capabilities, conduct experiments, adjust the company's behavior, without hesitate learn from previous failure and share experience with others; and finally, Company's ability to simplify the organizational structure. Strategy should be aimed at helping company to achieve its success due to a series of strategies that allow a company to separately decide its direction to the most prominent choices to create company's excellence. Hence, adaptability is considered as the ability of the company to form and implement a strategy that is most effectively in gaining company's excellence.

According to Reeves \& Deimler [2], there are four organizational capabilities that could create competitive advantage. Firstly, the ability to read and act on signs, which company required to identify the initial signs of change from its external business environment, and then understand and react quickly and radically if needed. Secondly, the ability to experiment new approaches and technologies which is enabling company to experiment with newer and updated phase to create, trial, and replicate a large amount of innovative ideas at full pace, lowered cost and risk than its competitors. Thirdly, the ability to manage a complex multi company systems which is related to the existence of company's barriers and changes in an industrial structure. A company needs to adapt its strategies to interconnected International industries. Lastly, the ability to mobilize the full potential or 
resources including human resources working in the organization, it would be related with more flexible structures, more flexible decision-making processes, and knowledge-sharing.

Referring to Girneata [9], a company that able to adapt well with its environmental changes could generates a positive effect to its functions. Therefore, there are some characteristics of company that able to adapt. Firstly, the company usually has a sloping structure whereas its goals are achieved by its members based on knowledge and skills, but not based on hierarchy. Secondly, most of the company's members clearly understand the goals and outcomes of the company and also committed to achieving its goals collectively which will also simultaneously achieve their personal goals. Thirdly, leaders together with their subordinates take part together in exchanging ideas to understand, seek for clarity and find out solution of organizational problems. New innovative ideas should emerge as the potential solutions required. Fourth, company could react quickly to existing business environmental influences. And lastly, company usually implements the adaptive learning system as a source if strategic excellence, in which company's members should be trained to be able to adapt and act quickly as well, through information sharing of each informed effectively.

\subsection{Competitive advantage}

Competitive advantage is considered as one of the terms which is in a vague and debatable concept in strategic management studies. As a key concept in strategic thinking, it aims to be able to differentiate the performance that occurs between existing companies. In terms of company growth, it can be juxtaposed as a long-term plan for a series of actions that enabling a company to achieve its expected performance goals. Competitive advantage is even dubbed as "a long-lived and conceptually troubled concept" [10], because of its longstanding concept that has been known but yet is still always a nature that debatable both by definition and overall implementation. However, it refers to the thought of Sigalas et al. [11] that considered competitive advantage as an exploitation of market opportunities and elimination of threats from competitors that could generate profits above overall industry's ability to generate.

\subsection{Social networking and adaptability}

Company that has a broad and strong networking will be able to support its combinative capabilities in distributing and integrating resources that also could be valuable to company itself. Thus, its resources will become a knowledge to be transformed and exploited into a better consideration for the next step [12]. Therefore, the wider a leader has a connection and is also accompanied by a strong connection, that leader could gather as much information as needed to decide the next step that company will take [12-14]. This thought could be a basis for creating a better network connection that a company has. With its high adaptability, a company could make it possible to adjust to its business environment so that the role of competitors could be repressed, or in a better scenario it is possible to change the role of competitors who are supposed to be rivals into friends. For example, is the role of government agency that could be an advantage for companies that have a good relationship. Hence, when a collaboration and good relationship are well established, there should be profits that benefited the company. However, before achieving these conditions, there are several important things to remember that government policies, for example, could hinder and restrain some company for its binding rules and policies, since it is considered as burden by choosing the right decision by complying or not.

A company with high adaptability could adjust to the rules or policies effectively, although it could be burdensome the company. However, as a result of compliance and 
mutual cooperation in other side, it is possible to form a good cooperation and relationship between the company and its potential collaborator. Therefore,

H1: Social networking has a significant effect toward adaptability.

\subsection{Adaptability and competitive advantage}

Company's adaptability toward its business environment has several terms, one of which is dynamism [15] which could be interpreted as an unpredictable condition that leads to the level of change and innovation in an industry and includes its consumers. In a dynamic business environment condition, resources are sometimes very difficult to obtain, coupled with the need for efficiency makes the need for adjustment and implementation become harder. Hence, it requires changes by the company to achieve short-term excellence. Nonetheless, company that able to adapt well or struggling with high adaptability will increase its competitiveness to survive and compete [16].

Reeves \& Deimler [2] argued their thought that related to competitive advantage, implicitly or explicitly, it is a factor that could achieve competitive advantage, especially in a dynamic condition and full of uncertainty.

Therefore, H2: Adaptability has a significant effect toward competitive advantage.

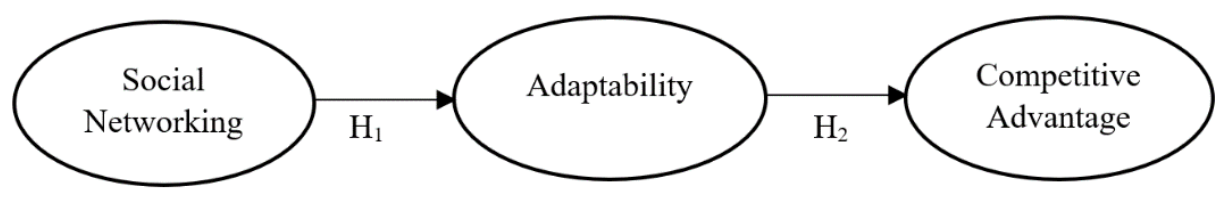

Fig. 1. Research model

\section{Method}

This is a quantitative research, whereas scientific methods used to obtain valid data with its aim of finding, proving, and developing knowledge to understand, solve, and anticipate problems in certain fields. This research also used explanatory approach which explains the relationship between variables, and to identify causal relationships between variables as represented and clearly defined in the research problems. Unit analysis in this study is restaurants in Surabaya city, East Java, Indonesia. The respondents will be the restaurant owners, due to their significant roles in determining the company's strategy and decision making. Thus, it is expected to be valid as a representative of the restaurants.

This study's sampling refers to non-probability sampling techniques, whereas each member in the population does not have the same opportunity to be chosen as a respondent, and also accompanied by purposive sampling approach which allows to determine the criteria required to be a respondent. The criteria include local independent restaurants that only have outlets in Surabaya city; and it has a maximum number of employees of 20 peoples.

Total sample that required for this study, refers to Hair et al. [17] with "10-times rule method" which stated that the required minimum sample for partial least square - structural equation modelling is ten times the maximum number of inner and outer model lines leading to latent variables. Since this study requires minimum 20 sample for respondents, the total sample for this study is 30 restaurants. 
Variables that used in this research include three main variables as referred to Social Networking (X1) as the exogenous variable; Adaptability (Y1) as the endogenous intervening variable; and Competitive Advantage (Y2) as the endogenous variable. This research uses 5-point Likert scale to measure the variables ranging from Strongly Disagree to Strongly Agree. The measurement for Social Networking referred to the adoption concept and measurement by Fernandez-Perez et al., [12] into three-item indicators including "Business owner's ability to establish a long term collaboration with business partners"; The measurement for Adaptability referred to the adoption concept by Reeves \& Deimler [2] and Girneata [9] into three-item indicators including "Ability to identify external business environment's changes"; Lastly, for Competitive Advantage referred to the adoption concept by Sigalas et al., [11] and Sigalas [10] into three-item indicators including "Producing product/service with competitive cost".

\section{Research and discussion}

Based on the result conducted by SmartPLS 2.0, validity testing can be seen in Table 1 which shows loading factor and cross loading as an evaluation of convergent and discriminant validity, which for loading factor shows that all loading values are above 0.5 which could be said that all the constructing indicators are valid. Related to discriminant validity, all constructing loading values have a higher correlation value to its construct than its correlation with the other constructs.

Table 1. Loading factor and cross loading factor

\begin{tabular}{|l|l|l|l|}
\hline & Adaptability & $\begin{array}{l}\text { Competitive } \\
\text { Advantage }\end{array}$ & $\begin{array}{l}\text { Social } \\
\text { Networking }\end{array}$ \\
\hline X11 & 0.1039 & -0.0036 & $\mathbf{0 . 7 4 3 2}$ \\
\hline X12 & 0.3143 & 0.1355 & $\mathbf{0 . 9 3 5 2}$ \\
\hline X13 & 0.4069 & -0.0972 & $\mathbf{0 . 9 4 7 9}$ \\
\hline Y11 & $\mathbf{0 . 8 9 4 6}$ & 0.2477 & 0.2382 \\
\hline Y12 & $\mathbf{0 . 9 1 9}$ & 0.1777 & 0.4074 \\
\hline Y13 & $\mathbf{0 . 8 8 9 8}$ & 0.0653 & 0.3094 \\
\hline Y21 & 0.0236 & $\mathbf{0 . 8 1 0 6}$ & -0.2671 \\
\hline Y22 & 0.1848 & $\mathbf{0 . 9 3 4}$ & -0.0112 \\
\hline Y23 & 0.1712 & $\mathbf{0 . 9 0 4}$ & 0.0561 \\
\hline
\end{tabular}

Based on the result for reliability testing, the result indicates that the composite reliability in each construct are 0.91 (Social Networking); 0.93 (Adaptability); and 0.92 (Competitive Advantage). Whereas Cronbach's Alpha shows a value of 0.87 (Social Networking); 0.89 (Adaptability); and 0.88 (Competitive Advantage). As a measurement of high reliability result, it is required that the value of each reliability higher than 0.7 . Therefore, all constructing indicators are considered as reliable. 


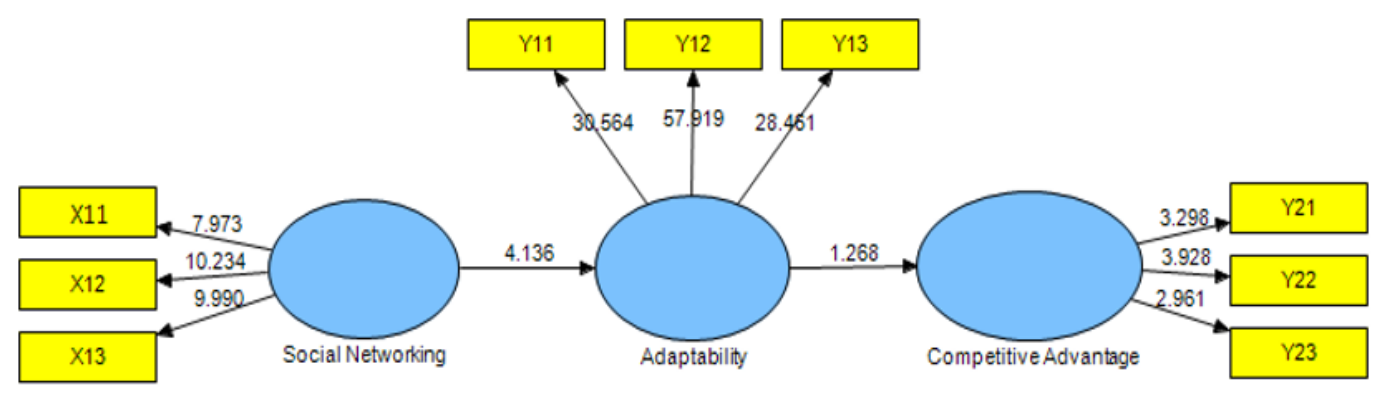

Fig. 2. Final research model.

Based on significance testing, it is shown that the T-Statistic value has a value that greater than 1.96 thus, it can be said that the effect between constructs is significant, conversely if the value is smaller than 1.96, the effect between constructs is not significant. Based on Table 2, it shows that the effect of Social Networking on Adaptability is significant; while the effect of Adaptability on Competitive Advantage is not significant. As the percentage effect of Social Networking on Adaptability could be explained at $13.2 \%$ while the remaining $86.8 \%$ is influenced by other factors; The percentage effect of Adaptability on Competitive Advantage could be explained at $3.4 \%$ while the remaining $96.6 \%$ is influenced by other factors.

Table 2. R Square and T Statistic Value

\begin{tabular}{|c|c|c|}
\hline & R Square & $\begin{array}{c}\text { T } \\
\text { Statistic }\end{array}$ \\
\hline Social Networking $\rightarrow$ Adaptability & 0.132 & 4.136 \\
\hline Adaptability $\rightarrow$ Competitive Advantage & 0.034 & 1.268 \\
\hline
\end{tabular}

Related to hypothesis testing, based on the result it is shown that Social Networking has a significant effect on Adaptability, therefore H1 is supported; while Adaptability has no significant effect on Competitive Advantage, therefore $\mathrm{H} 2$ is not supported.

In this study, shown that the leader's ability in developing networks could provide benefits that help to increase organizational capacity. The result of this study provides an empirical evidence in support of previous studies $[12,13]$. It refers that the broader leader's cooperation network which also supported by the intensity of good relationships will facilitate collaboration that occurs between organizations. Thus, it could provide benefits for organization to quickly adapt and adjust with changes, and also be able to survive the competition.

This capability is very useful for SMEs, especially to adjust against uncertainty within business environment and also against threat from stronger competitors. The result of the study also reinforces the opinion of Boss [18] states that organization often fail as a result of its leader's role who is unwilling or incapable to adapt and dealing with uncertainty, and it is obviously a leadership choice to remain current or adapt. 
The result of the study indicates organization's capability to adapt is still not enough to explain its effect on increasing Competitive Advantage. Hence, it is unable to empirically support the previous study by Armstrong [16], as well as the concept of thought proposed by Reeves \& Deimler [2]. Statistical argument shows that Adaptability's role in increasing Competitive Advantage is not significant. Thus, it could be considered to have no significant impact. Nonetheless, the result actually supports Sigalas' [11] that Competitive Advantage is a concept that is not easy to measure and justified, therefore it will always be debatable. It also proves that Competitive Advantage cannot be easily created based only on one type capability, instead of an accumulation of dynamic capabilities which could generate profits above average compared to competitors [13].

In SMEs' perspective, it could be said that having the capability to adapt is considered as inadequate to create the expected Competitive Advantage to compete. Despite of being sceptical, SMEs often have many weaknesses which are encouraged to not to compete with stronger companies [1]. However, by having the capability to adapt, it is expected that SMEs could strengthen their ability to survive with lesser expectations to compete equally with larger or stronger companies.

\section{Conclusion}

This is the initial study which investigate the role of Social Networking toward Adaptability and Competitive Advantage on SMEs in Indonesia. The result showed that Social Networking has a significant effect on Adaptability. However, Adaptability has no significant effect on Competitive Advantage.

As a suggestion for further research, it is expected to conduct empirical research on SMEs in Indonesia within various industries to provide a better in-depth understanding regarding the role of Social Networking and Adaptability in SMEs. It also suggests providing an adequate sufficient data in order to provide a better explanation.

\section{References}

1. J.A. Aragon-Correa, N. Hurtado-Torres, S. Sharma, V.J. Garcia-Morales, Journal of Environmental Management, 86:88-103(2008).

https://www.sciencedirect.com/science/article/pii/S0301479706003811

2. M. Reeves, M. Deimler, Harvard Business Review. 89:134-141(2011). https://hbr.org/2011/07/adaptability-the-new-competitive-advantage

3. D. Recklies, (2015). Adaptability - the new competitive advantage. [Online] from http://www.themanager.org/2015/01/adaptability-new-competitive-advantage/ (2015). [Accessed on April 2019].

4. L.Q. Wei, C.M. Lau, Human Relations. 63,10:1487-1511 (2010). https://journals.sagepub.com/doi/abs/10.1177/0018726709359720.

5. J. Steinfeld, Build to survive: the darwin approach to business. [Online] from http://www.inc.com/jay-steinfeld/darwin-approach-to-business.html(2011). [Accessed on April 2019].

6. Jawa Pos, (2014). MEA, antisipasi serbuan resto asing. [MEA, anticipate the invasion of foreign restaurants]. [Online] from

http://www2.jawapos.com/baca/artikel/10695/mea-antisipasi-serbuan-resto-asing (2014). [Accessed on April 2019]. [in Bahasa Indonesia].

7. S. Gronum, M. Verreynne, T. Kastelle, Journal of Small Business Management. 50,2:257-282(2012). https://onlinelibrary.wiley.com/doi/abs/10.1111/j.1540627X.2012.00353.X. 
8. D. Ford, S. Mouzas, Industrial Marketing Management. 42:433-442(2013). https://www.sciencedirect.com/science/article/pii/S001985011300031X.

9. A. Girneata, Economic Questions, Issues and Problems, 234-249(2014). https://www.researchgate.net/profile/Adriana_Dima/publication/299092036_Adaptabi lity -

A_Strategic_Capability_During_Crisis/links/56eeb6ac08ae4b8b5e751dd2/Adaptabili ty-A-Strategic-Capability-During-Crisis.pdf.

10. C. Sigalas, Decision. 53,9:2004-2016(2015).

https://www.emeraldinsight.com/doi/full/10.1108/MD-05-2015-0185.

11. C. Sigalas, V. Pekka-Economou, N.B. Georgopoulos, Journal of Strategy and Management. 6,4:320-342(2013).

https://www.emeraldinsight.com/doi/abs/10.1108/JSMA-03-2013-0015.

12. V. Fernandez-Perez, V.J. Garcia-Morales, O.F. Bustinza-Sanchez. Personnel Review. 41,6:777-812(2012).

https://www.emeraldinsight.com/doi/abs/10.1108/00483481211263719

13. V. Fernandez-Perez, A.J. Verdu-Jover, J. Benitez-Amado, Personnel Review. 42,2 :134-153(2013).

https://www.emeraldinsight.com/doi/abs/10.1108/00483481311309357.

14. V. Fernandez-Perez, F.J.L. Montes, V.J. Garcia-Morales, Industrial Management \& Data Systems. 114,6:858-871(2014).

https://www.emerald.com/insight/content/doi/10.1108/IMDS-11-2013-0483/full/html.

15. D. Li, J. Liu, Journal of Business Research. 67:2793-2799(2014). https://www.sciencedirect.com/science/article/pii/S0148296312002202.

16. C.E. Armstrong, Journal of Strategic Management. 6,4:377-398(2013). https://www.emeraldinsight.com/doi/abs/10.1108/JSMA-06-2012-0034.

17. J.F. Hair, M. Sarstedt, C.M. Ringle, J.A. Mena, Journal of the Academic Marketing Science. 40:414-433(2012). https://link.springer.com/article/10.1007/s11747-0110261-6.

18. Boss, J. Staying competitive requires adaptability. [Online] from https://www.forbes.com/sites/jeffboss/2016/04/26/staying-competitive-requiresadaptability/\#36ac9ff7e6f9 (2016). [Accessed on April 2019]. 